\title{
Trabalho interprofissional e as Práticas Integrativas e Complementares no contexto da Atenção Primária à Saúde: potenciais e desafios
}

\author{
Interprofessional work in Integrative and Complementary Practices \\ in the context of Primary Health Care: potentials and challenges
}

Nelson Filice de Barros' ${ }^{\mathbf{1}}$ Cristiane Spadacio $\mathbf{2}$, Marcelo Viana da Costa ${ }^{\mathbf{3}}$

DOI: 10.1590/0103-11042018S111

1 Universidade Estadual de Campinas (Unicamp), Faculdade de Ciências Médicas, Laboratório de Práticas Alternativas,

Complementares e Integrativas em Saúde (Lapacis) - Campinas (SP) Brasil.

Orcid: https://orcid org/0000-0002-23890056

nelfel@uol.com.br

2 Universidade Estadual de Campinas (Unicamp), Faculdade de Ciências Médicas, Laboratório de Práticas Alternativas, Complementares e Integrativas em Saúde (Lapacis) - Campinas (SP), Brasil. Universidade Federal do Rio Grande do Norte (UFRN), Escola Multicampi de Ciências

Médicas (EMCM) - Natal (RN), Brasil.

Orcid: https://orcid org/0000-0002-4582-

3540

cris.spadacio@gmail.com

3 Universidade Federal do Rio Grande do Norte (UFRN), Escola Multicampi de Ciências Médicas (EMCM), Grupo Interdisciplinar de Pesquisa em Educação, Saúde e

Sociedade (Gipess) - Natal (RN), Brasil.

Orcid: https://orcid

org/0000-0002-3673-

2727

vianacostam@yahoo.com.br
RESUMO No campo da saúde no Brasil, o trabalho em equipe no contexto da Atenção Primária à Saúde (APS) se configura como estratégia para avançar na perspectiva do cuidado integral, bem como para ampliar a resolutividade nos demais níveis de atenção no Sistema Único de Saúde (SUS). Este artigo objetivou analisar os potenciais e desafios do trabalho interprofissional com as Práticas Integrativas e Complementares (PIC) no contexto da APS. Estudo de abordagem qualitativa, efetuado no período de setembro de 2014 a junho de 2015. Foram realizadas entrevistas semiestruturadas por telefone com os coordenadores das Unidades Básicas de Saúde para mapear a ofertas das PIC. Os resultados foram submetidos à análise de conteúdo temática, revelando oportunidades e entraves para o trabalho interprofissional no contexto da APS. Observou-se que, em muitas experiências, o trabalho em equipe não se fundamenta na colaboração. Por outro lado, as PIC promovem maior satisfação no trabalho em razão das mudanças que promove na organização do trabalho, por meio da maior interação entre práticas e saberes dos profissionais e entres estes e os usuários.

PALAVRAS-CHAVE Atenção Primária à Saúde. Equipe de assistência ao paciente. Terapias complementares. Sistema Único de Saúde.

ABSTRACT In the field of health in Brazil, teamwork in the context of Primary Health Care (PHC) is a strategy to advance the perspective of comprehensive healthcare. It is also a way to increase the effectiveness of care in the other levels of attention in the Unified Health System (SUS). The objective of this article is to analyze the potentials and the challenges of interprofessional work with Integrative and Complementary Practices (PIC) in the context of PHC. A qualitative study was carried out from September 2014 to June 2015. Semi-structured telephone interviews were conducted with the coordinators of the services Basic Health Units to map out the PIC offer. The results were submitted to thematic content analysis and revealed opportunities and obstacles for interprofessional work in the context of PHC. It was observed that, in many experiences, teamwork is not based on collaboration. On the other hand, PIC promote greater job satisfaction due to the changes that it promotes in the organization of work, through the greater interaction between practices and knowledges of professionals and between professionals and users.

KEYWORDS Primary Health Care. Patient care team. Complementary therapies. Unified Health System. 


\section{Introdução}

No campo da saúde no Brasil, o trabalho em equipe configura-se como estratégia de cuidado integral em todos os níveis de atenção do Sistema Único de Saúde (SUS), em especial, na Atenção Primária à Saúde (APS). É considerado como um dos pilares do trabalho em saúde e toma as necessidades de saúde como objeto multidimensional que engloba aspectos biológicos, psicológicos, sociais, políticos, econômicos e culturais.

Na APS, a Estratégia Saúde da Família (ESF) busca (res)situar o usuário, a família e a comunidade no centro do processo de cuidado, o que exige uma intrínseca interação entre as diferentes categorias profissionais que compõem as equipes de saúde. Existe certa confusão conceitual entre trabalho multiprofissional, trabalho em equipe, trabalho interprofissional e prática colaborativa. O nível de interação, o compartilhamento de objetivos em comum, a identidade de equipe e a centralidade no usuário e suas necessidades são aspectos centrais para a diferenciação desses termos ${ }^{\mathbf{1}, \mathbf{2}}$.

A perspectiva interprofissional é bastante distinta da multiprofissional. Enquanto esta relaciona-se com o trabalho em equipes constituídas por diferentes profissionais, sem a necessária interação entre os membros e, por vezes, apenas dividindo o mesmo espaço, com limitada ou nenhuma interação; aquela compartilha objetivos, desenvolve identidade de equipe $\mathrm{e}^{3}$ busca o cuidado integral, levando em consideração o caráter complexo e dinâmico das necessidades de saúde de indivíduos e coletivos, considerados coprodutores dos atos em saúde ${ }^{4}$.

Destarte, é preciso transitar do trabalho em paralelo (multiprofissional) para o articulado e integrado (interprofissional), orientado pelas necessidades de saúde ${ }^{5}$. Esse trânsito se justifica pela necessidade de superar o contexto marcado pelo trabalho fortemente parcelado e centrado no profissional. Em outras palavras, é necessário passar da equipe como agrupamento de agentes, para a equipe como integração de trabalhos ${ }^{6}$. Na primeira noção, a tônica do trabalho é a fragmentação e a justaposição de ações; na segunda, evidencia-se a integralidade das ações de saúde. $O$ fortalecimento dessa passagem deve fazer parte das preocupações cotidianas do trabalho, para que os profissionais articulem as decisões e configurem

[...] na relação recíproca entre as múltiplas intervenções técnicas e a interação dos agentes de diferentes áreas profissionais [...] [o] trabalho em equipe multiprofissional [como] uma modalidade de trabalho coletivo ${ }^{6(108)}$.

A integração de trabalhos pela equipe, em oposição ao agrupamento de agentes, é especialmente importante para refletir sobre o trabalho interprofissional com as Práticas Integrativas e Complementares (PIC) na APS. A discussão sobre inclusão de PIC no sistema nacional de saúde brasileiro teve início na década de 1980, com destaque para a VIII Conferência Nacional de Saúde, em 1986, e após a criação do SUS, em 1988. Com a descentralização e a participação popular, os estados e municípios ganharam maior autonomia na definição de suas políticas e ações em saúde, contexto no qual foram desenvolvidas algumas experiências pioneiras e que deram origem às reivindicações para regulamentação das PIC em nível nacional7.

As iniciativas existentes ocorriam sem ter diretrizes a serem seguidas, colaborando para o desenvolvimento

de modo desigual, descontinuado e, muitas vezes, sem o devido registro, fornecimento adequado de insumos ou ações de acompanhamento e avaliação8(4).

A partir de 2006, a oferta da homeopatia, da medicina tradicional chinesa/acupuntura, da medicina antroposófica, da fitoterapia/plantas medicinais e do termalismo/crenoterapia é regulamenta e definida pela Política Nacional de Práticas Integrativas e Complementares (PNPIC) no SUS. 
Ao longo desses 12 anos de existência da PNPIC, tem sido investigado o processo de implantação das diferentes práticas e do seu potencial de cuidado no cotidiano dos serviços de $\mathrm{APS}^{9-12}$. Contudo, nenhuma investigação analisou a integração de trabalhos em oposição ao agrupamento de agentes na APS tendo como eixo as PIC. Assim, o presente artigo tem como objetivo analisar os potenciais e desafios do trabalho interprofissional com as PIC no contexto da APS.

\section{Material e métodos}

Este artigo analisa resultados do primeiro de três estudos que integram o projeto 'As Racionalidades Médicas e Práticas Integrativas e Complementares nos serviços de APS na Região Metropolitana de Campinas/SP', desenvolvido pelo Laboratório de Práticas Alternativas, Complementares e Integrativas em Saúde (Lapacis/Unicamp), o qual tem como parte de sua missão apoiar ações governamentais de implementação das PIC no SUS.

No período de setembro de 2014 a junho de 2015, após aprovação do projeto pelo Comitê de Ética e pelo Colegiado de Gestão Regional da Região Metropolitana de Campinas, desenvolveu-se um censo em todos os serviços de APS dos 20 municípios da Região Metropolitana de Campinas (RMC), para identificar quais eram as PIC ofertadas e quais as avaliações dos coordenadores das unidades sobre o uso dessas práticas no serviço.

Inicialmente, realizou-se levantamento do número de Unidades Básicas de Saúde (UBS) no Observatório de Indicadores da RMC. Após checagem do número de unidades no Cadastro Nacional de Estabelecimentos de Saúde (CNES), identificou-se um total de 285 UBS a serem investigadas. Foram realizadas entrevistas semiestruturadas por telefone com os coordenadores das UBS e identificadas 117 que ofertavam alguma PIC naquele momento, as quais estavam concentradas em 8 dos $20 \mathrm{mu}$ nicípios da RMC.
O roteiro de entrevista continha, entre outras, as seguintes perguntas: 'Quais os aspectos negativos do desenvolvimento das PIC na APS para a equipe? Por quê?' 'Quais os aspectos positivos do desenvolvimento das PIC na APS para a equipe? Por quê?' As respostas foram gravadas, transcritas e submetidas à análise temática de conteúdo, a partir do referencial da sociologia da saúde e saúde coletiva, em busca de constituir uma sociologia das PIC.

A estrutura de análise qualitativa dos dados obedeceu a duas etapas principais. A primeira delas foi a 'pré-análise', em que se realizou a leitura de todas as entrevistas para a compreensão global do contexto. Após essa primeira leitura, foram identificados os núcleos de sentidos, sempre elencando extratos de falas que representavam, de fato, os argumentos mais significativos para essa fase. Esse é um processo conhecido como 'load your mind', na busca por padrões, relações e repetições no corpus global das entrevistas - aqui as categorias analíticas iniciais já começam a se delinear. $\mathrm{Na}$ segunda etapa, as categorias analíticas foram estabelecidas, e recategorizações aconteceram, sempre buscando um nível interpretativo dos discursos dos entrevistados.

As categorias analíticas emergidas dos núcleos de sentidos positivos e negativos foram as seguintes: i) integração de diferentes profissões que compõem a equipe; ii) estratégia de aproximação de profissionais e das equipes com usuários; iii) dispositivo de prevenção e promoção da saúde para a equipe; iv) conflito com outras atividades da equipe; $v$ ) sobrecarga de trabalho dos profissionais que conduzem as PIC; vi) atividade não prioritária na rotina de atendimento. Na apresentação dos resultados, conforme recomenda Minayo, "as categorias empíricas passaram por um novo processo de teorização a partir do referencial adotado"13(625)

\section{Resultados e discussão}

As PIC são um bom analisador do processo de trabalho em saúde na APS, ou seja, 
são 'boas para pensar' as relações de trabalho entre profissionais no contexto do SUS. Cabe, contudo, antes de aprofundar essa reflexão, apresentar o perfil dos entrevistados, que exibiram as seguintes características: predominantemente coordenadores dos serviços $(\mathrm{N}=102 / 87,0 \%)$, dos quais $89,7 \%(\mathrm{~N}=105)$ eram do sexo feminino, com idade média de 41,45 anos; $76,1 \%(\mathrm{~N}=89)$ se referenciou de etnicidade branca; os maiores níveis de escolaridades referidos foram pós-graduação latu sensu (especialização) e superior completo, com $47,9 \%$ e $40,2 \%$ respectivamente; a religião católica predominou com $55 \%$ dos relatos; a profissão mais frequente foi de enfermeiros (70,4\%), seguida por odontólogos (9,6\%) e médicos (8,7\%); o tipo de vínculo da maior parte dos entrevistados é direto com o município, para atuar no SUS local (98,3\%); e o tempo médio de vínculo com a prefeitura, de 132,15 meses, enquanto com a UBS foi de 55,58 meses.

Os profissionais entrevistados, coordenadores de serviços que ofertam alguma PIC em 8 dos 20 municípios da RMC, atribuíram sentidos positivos à oferta de PIC para a equipe fazendo emergir as seguintes categorias analíticas:

i) 'integração de diferentes profissões que compõem a equipe': as PIC constituem-se como estratégias de integração entre as diferentes profissões que compõem as equipes de APS, uma vez que a presença dessas práticas nos serviços é capaz de gerar, segundo um dos entrevistados:

[...] um espirito de equipe, eu acho que a equipe melhora a atenção, com mais solidariedade... melhora a questão da amizade entre a equipe, até para o desenvolvimento do trabalho. (entrevistado 74).

ii) 'estratégia de aproximação de profissionais e das equipes com usuários', delineando-se como atividades prazerosas que aproximam os profissionais das equipes com usuários e cuidadores da APS, tal como expresso no seguinte discurso:
Eu acho que quando a gente consegue proporcionar num centro de saúde atividades prazerosas tanto pro profissional, mas que também possa melhorar o vínculo com a comunidade, eu acho que sempre traz pra gente um benefício importante. (entrevistado 268).

iii) 'dispositivo de prevenção e promoção da saúde para a equipe', na qual as PIC apresentam, para a equipe, uma possibilidade para o trabalho de prevenção e promoção da saúde. Afirmou um dos entrevistados que

A equipe se sente assim, trabalhando numa missão mais preventiva quando trabalha com as práticas integrativas... então nesse espaço é um espaço também de produzir saúde. (entrevistado 246).

Os aspectos positivos do desenvolvimento das PIC para a equipe, atribuídos pelos coordenadores de serviços, constituem, portanto, uma estratégia de integração profissional e práticas de cuidado para o trabalho das equipes na APS. Observou-se uma relação estreita entre estes sentidos positivos das PIC e a experiência de equipes que integram o trabalho ${ }^{6}$ na APS. Assim, identificou-se forte semelhança entre as PIC como estratégias de integração entre as diferentes profissões e a formação de conexões e interfaces entre os profissionais; articulação de práticas e saberes no enfrentamento de cada situação identificada; construção da alteridade entre os profissionais; superação da inércia e indiferença burocrática; e reconhecimento e respeito aos diferentes conhecimentos.

Observou-se, também, forte afinidade entre as PIC como atividades prazerosas que aproximam os profissionais com usuários e a integração entre os profissionais com o intuito de tomar a conduta adequada de acordo com cada necessidade; estreitamento do vínculo com a comunidade e entre os próprios profissionais da equipe. Por fim, identificaram-se as PIC como uma perspectiva de prevenção e produção da saúde com 
a recomposição de processos de trabalhos distintos; a quebra da divisão do processo de trabalho; corresponsabilização pelos problemas apresentados à equipe; e o estímulo ao trabalho partilhado.

A implantação das PIC na APS alcança a dimensão de uma inovação técnico-tecnológica que rearranja a organização e o processo de trabalho. Com elas, os entrevistados relataram mudanças substanciais do ponto de vista da qualidade do trabalho, pois introduziram uma dimensão prazerosa ao labor do campo da saúde, assim como desenvolveram uma virada paradigmática da patogênese para a prevenção e promoção da saúde. Não bastassem essas mudanças internas do processo de trabalho, também trouxeram mudanças da organização do trabalho ao propiciar maior integração e vinculação dos profissionais das equipes com as populações atendidas.

Nas entrevistas, foi possível perceber que o elemento principal da mudança da dinâmica do trabalho em saúde com as PIC foi a centralização do cuidado no usuário e suas necessidades. Assim, observou-se um deslocamento da perspectiva centrada no procedimento ou no profissional, para uma ordenação a partir das necessidades dos usuários. As PIC se apresentaram como mecanismo capaz de desvendar as potências do trabalho interprofissional, com claro benefícios aos usuários, que se sentiram mais 'atendidos' tendo em vista as novas relações que foram estabelecidas entre eles e os profissionais. Também para os profissionais de saúde houve mudança significativa, por não estarem a todo momento no campo da atenção curativista e individual. Além disso, as PIC foram identificadas pelos entrevistados como formas de estabelecer a horizontalização dos poderes, em detrimento das relações verticalizadas no processo de trabalho das equipes, que é uma das maiores barreiras da interprofissionalidade. A vivência de maior equilíbrio nas relações de poder favoreceu a comunicação, a tomada de decisões compartilhadas, a escuta atenta e respeitosa e uma dinâmica permanente de aprender com o outro, sobre o outro e para o outro 4 .

No entanto, os coordenadores de serviços de saúde da APS também identificaram um conjunto de aspetos negativos da oferta de PIC para as equipes de saúde. De seus discursos, emergiram as seguintes categorias:

iv) 'conflitos com outras atividades da equipe', no sentido de que as PIC gerariam tensionamentos para a realização das demais atividades, como observa-se no trecho abaixo:

a única questão é assim, que como elas [funcionárias] estão alocadas no centro de saúde, elas têm inúmeras atribuições né, então a gente só tem que casar com todas as atividades que elas têm que fazer também, para que elas mantenham o grupo, que a gente avalia como superimportante, mas não deixem de fazer outras funções que também são importantes, né? (245).

v) 'sobrecarga de trabalho dos profissionais que conduzem as PIC', uma vez que há pouco recurso humano e de estrutura para a realização das atividades básicas do processo de trabalho dos profissionais. Afirmou um dos coordenadores que

Pra equipe, olha, o negativo que eu vejo é que a gente tem pouco recurso para dar conta do trabalho e saindo pessoas para fazer uma coisa fora, eles tomam isto como negativo. Por exemplo, eu estou aqui no curativo, tenho 15 pacientes para atender de manhã, e quem está escalado comigo sai duas horas da unidade para fazer este grupo fora. Quando a gente está com um recurso humano bem compativel eu vejo que não tem tanto conflito, entendeu? É a única coisa que eu vejo de negativo. (281).

vi) 'atividade não prioritária na rotina de atendimento' da equipe no serviço, como exposto na fala abaixo:

Às vezes eu acho que a maior dificuldade que a gente tem hoje é que com o número pequeno de profissionais que a gente tem, a falta de $\mathrm{RH}$, 
muitas vezes a gente acaba redirecionando 0 tempo, a carga horária destes profissionais para as atividades que são a demanda principal da unidade, e aí as Práticas acabam não sendo priorizadas, neste contexto... por causa das dificuldades que a gente tem de liberar... (272).

Estes três aspectos negativos são importantes para sinalizar conflitos de baixo reconhecimento e visibilidade nas relações internas das equipes de saúde. Na medida em que um profissional da equipe se interessar por alguma PIC e fizer o investimento de formar-se para ofertá-la no serviço, terá que negociar algumas horas com os outros profissionais da equipe. Todo esse processo deveria fazer parte do trabalho da equipe, no entanto, como narraram os coordenadores, a oferta desse tipo de prática na APS não apenas gera conflito e sobrecarga aos profissionais da equipe, como também não faz parte das 'atividades que são a demanda principal da unidade'.

De outra maneira, é possível afirmar, a partir de um exercício de generalização analítica posicionado nas PIC e que alcança muitas outras práticas no campo da saúde, que o profissional de uma equipe que se interesse por alguma prática de cuidado não protocolar e hegemônica do campo da saúde, ou que busque inovar a organização do trabalho nos serviços da APS, sofrerá diferentes formas de constrangimento pelos outros profissionais que compõem a equipe multiprofissional.

Pode ser que a ação inovadora do profissional seja a expressão de uma formação especializada e orientada pelo modelo biomédico hegemônico, majoritariamente apoiado na lógica assistencialista e fragmentária, que dificulta trabalho em equipe. Todavia, pode ser, também, que o interesse de um profissional pelo 'novo' seja a expressão genuína do desejo de romper com a inércia institucional e construir um modelo de cuidado, não apenas integral, mas também emancipatório. Pode ser, ainda, que o interesse por introduzir novas práticas na equipe seja uma mistura entre individualismo e compromisso com o cuidado da população na APS. Entretanto, qualquer que seja a motivação do profissional, sua ação resultará em possíveis conflitos interprofissionais com os outros membros da equipe. Nessas tensões, estarão operando as relações de poder corporativas e estruturais do campo da saúde, as quais podem ser condicionantes da construção social e manutenção de assimetrias no trabalho das equipes de saúde.

No discurso de um coordenador, bastante semelhante ao de muitos outros entrevistados, observa-se a expressão desse complexo de relações de poder corporativo e estrutural nas equipes da APS em torno do não reconhecimento das PIC pelos profissionais não envolvidos com elas. Em suas palavras:

Eu acho que ainda não tem uma percepção definida pelos profissionais, pra complementar o modelo de 'consultação'. Pra mim é evidente isso [...] eu acho que pra equipe como um todo, mesmo envolvendo o profissional médico, ainda não caiu a ficha. Eu acho que a gente tem ainda pouco investimento nesta coisa de definir que é um modelo importante, uma opção importante para os profissionais e para o usuário, ainda tá... pouco investido. (entrevistado 240).

Claramente, mas de forma não linear, é possível ver expressas nesse discurso as relações hierarquizadas que permeiam o trabalho das equipes. Elas estão condicionadas, como vimos, à forma de organização do trabalho e, muitas vezes, também à hegemonia do poder técnico e político de uma categoria profissional. A assimetria dessas relações estabelece hierarquias, verticalizações e preconceitos, nas relações dos trabalhadores das equipes, podendo operar como obstáculo para o trabalho coletivo se concretizar, tornando-o fragmentado e desconectado ${ }^{14}$.

A potência do trabalho interprofissional no processo de reorientação do modelo de atenção à saúde não se sustenta apenas pelas 
iniciativas individuais. Nas falas anteriores, está clara a necessidade de maior apoio institucional para o fortalecimento do trabalho integrado e colaborativo, isso porque as normativas institucionais, algumas vezes, legitimam as disparidades de poder e não investem em novos arranjos de trabalho em saúde, que assegurem o efetivo trabalho em equipe. $\mathrm{O}$ debate sobre trabalho interprofissional precisa vir acompanhado de um movimento de reconhecimento e valorização de todos os profissionais. Enquanto as relações de poder e as formas de manutenção da desigualdade de poder não forem enfrentadas nas esferas macro, meso e microssocial, teremos dificuldades de avançar em uma cultura colaborativa ${ }^{15}$.

São muitas as implicações da assimetria de poder no interior da APS; e, possivelmente, a mais perversa é a de culpabilização das vítimas do processo. Diferentes eventos produzem a desmotivação dos profissionais para oferecem as PIC nos serviços de APS, que julgamos ser semelhante a processos de introdução de outras práticas não hegemônicas. Por um lado, há inadequação estrutural das unidades para o desenvolvimento do trabalho com técnicas de cuidado coletivas, como o Lian Gong que é uma das PIC coletiva de origem da Medicina Tradicional Chinesa. Por outro, ocorre a culpabilização dos usuários de "não se apossarem do grupo da maneira como deveria" (274), que por extensão poderá atingir o profissional responsável pelo grupo.

Todo esse processo pode ser relacionado, de maneira recorrente, com a introdução de novas práticas no processo de trabalho das equipes da APS. No caso de práticas corporais coletivas, a dificuldade tem início na arquitetura das UBS. Elas foram construídas tomando como referência o modelo de atenção centrado no atendimento clínico, com consultórios pequenos e sem espaços adequados para atividades de grupo. Nas UBS investigadas, quando essas práticas são ofertadas, exige-se improvisação em áreas externas, ou a utilização de outros equipamentos públicos e comunitários no entorno. Como se viu, pesa aos profissionais encarregados pela PIC adequar o espaço para o desenvolvimento da atividade; com isso, o profissional 'ausenta-se' do serviço, e o ciclo vicioso nas relações entre os profissionais da equipe é reforçado, com tensionamentos contínuos entre a oferta de PIC em relação à manutenção do funcionamento do atendimento da unidade e o desenvolvimento de PIC em relação às demandas principais dos serviços.

São notórios os relatos sobre a fragilidade da construção do modelo de trabalho interprofissional e as PIC na APS, com seus sentidos positivos e negativos atribuídos pelos coordenadores dos serviços, são um bom analisador das potencialidades e desafios do trabalho das equipes de saúde no SUS. Os resultados da nossa investigação sobre as PIC visibilizaram aspectos pouco considerados nos conflitos interprofissionais da organização e processo de trabalho das equipes, como, por exemplo, a insuficiência de condições estruturais, como falta de materiais, falta de funcionários e os baixos salários ${ }^{16,17}$.

Os diversos tipos de contratos de trabalho, tais como celetista (Consolidação das Leis do Trabalho - CLT), temporários e até indicação político-partidária em alguns casos, manifestam-se como problema para a consolidação do trabalho interprofissional. Tal situação gera instabilidades, dificultando efetivamente a criação de vínculo do profissional com o serviço, com a equipe e com a população atendida, enfraquecendo a lógica da interprofissionalidade na dinâmica do trabalho em saúde, em razão da alta rotatividade de seus membros, interferindo principalmente na identidade de equipe. A forma de contrato de trabalho interfere diretamente nos serviços prestados ${ }^{18}$, e as questões de remuneração econômica desigual para os profissionais das equipes são marcadores de profundas desigualdades ${ }^{19}$.

Com as PIC na APS, observou-se a assimetria de poder interprofissional, quase 
sempre estabelecida e mantida pelas relações corporativas, estrutura estatal e hierarquias sociais, que garantem privilégios econômicos e contratuais a determinados categorias profissionais. As relações hierarquizadas decorrentes dessa forma de organização do trabalho atravessam as equipes e, muitas vezes, dão-se como obstáculo para o trabalho coletivo ${ }^{16}$.

Há o predomínio da hegemonia do poder técnico e político dos profissionais médicos, que entram em conflito com outros profissionais de nível universitário e médio. $\mathrm{O}$ trabalho acaba sendo feito de forma fragmentada e desconectada da finalidade de seu objetivo fina: o cuidado integral das pessoas e suas necessidades. Para os médicos, regularmente, a equipe se resume à equipe médica, e a execução do trabalho interprofissional é dificultada pela falta de espaço dos 'não médicos' para o exercício autônomo de suas funções ${ }^{20}$. Nesse sentido, é importante ressaltar que a estruturação do trabalho em equipes multiprofissionais, por si só, não garante uma ruptura com a dinâmica das relações de poder médico-centrada. Por isso, as relações de poder no processo de trabalho interprofissional devem ser problematizadas como condição fundamental para o efetivo trabalho de colaboração das equipes multiprofissionais. Sobretudo, porque elas condicionam os diferentes estilos de gestão das equipes de saúde e a organização da integralidade do cuidado ${ }^{21}$.

Apesar das dificuldades evidenciadas, é preciso destacar os avanços que a composição de equipes multiprofissionais traz para a realidade dos serviços de APS. A proposta da ESF desenvolveu benefícios notáveis no trabalho em equipe com a corresponsabilização dos serviços, cogestão dos problemas e a superação da inércia e indiferença burocrática, que muitas vezes se apresentam como um entrave para o desenvolvimento do SUS. Com a perspectiva da ESF na APS, aconteceu, então, a recomposição de processos de trabalhos e a formação de conexões e interfaces entre as intervenções peculiares de cada área profissional, preservando as respectivas especificidades ${ }^{\mathbf{2 0} 21}$. Um outro avanço importante foi a construção da alteridade entre os profissionais, ou seja, o reconhecimento e o respeito ao outro como um estímulo ao trabalho partilhado, que são, muitas vezes, invisíveis e não quantificáveis em termos de indicadores ${ }^{16}$.

O desafio para as equipes multiprofissionais, no entanto, é de estabelecer fluxos de comunicação interpessoais que possibilitem a discussão dos problemas cotidianos, com liberdade para todos participarem das decisões. As atitudes de respeito, linguagem comum, disposição para aprender, corresponsabilização e união fazem parte dos desafios comunicacionais das equipe ${ }^{22}$. $\mathrm{O}$ trabalho realizado de forma conjunta e articulada exige comunicação fluida e permanente, sendo, inclusive, a condição para que as equipes de saúde ampliem sua capacidade de cuidado e de resolução integral dos problemas de saúde ${ }^{23}$.

A presença das PIC na APS mostrou a potencialidade de articular desafios cotidianos das equipes multiprofissionais com o desenvolvimento de trabalhos prazerosos para os profissionais e para as populações atendidas. Este aspecto aponta para o fortalecimento do trabalho interprofissional e das práticas colaborativas, situando a comunidade e os usuários na centralidade do processo de produção dos serviços de saúde.

\section{Conclusões}

A experiência de trabalho interprofissional nos serviços de saúde na APS apresenta-se, em princípio, como fácil de ser operacionalizada, porém, desenvolver a colaboração e a integração entre diversos núcleos de saberes e práticas é constantemente um desafio posto para os profissionais de saúde.

Fazem-se necessárias certas alterações da dinâmica do trabalho em saúde e nos fazeres 
do cotidiano de cada profissional. Há que se identificar nas equipes os elementos que configuram uma nova lógica no agir desses profissionais e na forma como se produz o cuidado em saúde ${ }^{20}$. Garantir que os profissionais estejam alinhados com a lógica de cuidado integral exige um esforço para além de suas formações acadêmicas básicas, sendo necessário o desenvolvimento de competências colaborativas para a superação de um labor individual e hierarquizado no interior dos serviços. Tais mudanças não se dão de maneira rápida, pois os profissionais que compõem as equipes foram formados em uma lógica de predomínio do poder do nível superior sobre o nível médio e da categoria médica sobre as demais. Nesse contexto, relações de poder hierarquizadas, estabelecidas entre os profissionais, configuram elementos que fortalecem a situação de assimetria de algumas profissões sobre outras, garantindo posições desiguais na equipe e nos serviços de saúde.

A mudança do paradigma que reforça a formação orientada para o labor individual, para a superespecialização e para as relações hierarquizadas exige diversas ações e posicionamentos dos próprios profissionais no nível macro, meso e microssocial24. Trabalhar em equipe demanda relações efetivas, com ênfase na comunicação, compartilhamento, parceria, interdependência e equilíbrio de poderes. Depreende-se, assim, a necessidade de constantes investimentos nas relações entre os membros da equipe, oportunizados pela oferta das PIC nos serviços.
Existem experiências positivas e negativas na organização do processo de trabalho de equipe interprofissional em saúde no Brasil. Oportunidades e entraves são observados em vários aspectos; e, neste artigo, foram problematizados por meio da análise das PIC nos serviços de APS dos municípios da RMC/SP. A partir desse evento, observamos que o cuidado realizado pelas equipes não tem sido desenvolvido em todo o seu potencial e que o contexto atual de significativas perdas no SUS pode dificultar ainda mais o desenvolvimento da lógica interprofissional e colaborativa.

\section{Colaboradores}

Barros NF contribuiu para elaboração do artigo com as seguintes atividades: concepção e o planejamento para a análise e a interpretação dos dados; contribuição na elaboração do rascunho; e participação da aprovação da versão final do manuscrito. Spadacio C contribuiu para elaboração do artigo com as seguintes atividades: concepção e o planejamento para a análise e a interpretação dos dados; contribuição na elaboração do rascunho; e participação da aprovação da versão final do manuscrito. Costa MV contribuiu para elaboração do artigo com as seguintes atividades: concepção e o planejamento para a análise e a interpretação dos dados; contribuição na elaboração do rascunho; e participação da aprovação da versão final do manuscrito. 


\section{Referências}

1. Goldman J, Zwarentein M, Bhattacharyya O, et al. Improving the clarity of the interprofessional field: implications for research and continuing interprofessional education. J. Contin. Educ. Health. Prof. [internet]. 2009 [acesso em 2018 out 9]; 29(3):151156. Disponível em: https://www.ncbi.nlm.nih.gov/ pubmed/19728378.

2. D'amour D, Ferrada-Videla M, San Martin Rodriguez L, et al. The conceptual basis for interprofessional collaboration: core concepts and theoretical frameworks. J. Interprof. Care. London. 2005 jul 19(sup1):116-131.

3. Agreli HF, Peuzzi M, Silva MC. Atenção centrada no paciente na prática interprofissional colaborativa. Inter. Com. Saúde. Edu. São Paulo 2017 maio 20(59):905-916.

4. Barr H, Koppel I, Reeves S, et al. Effective Interprofessional Education: Argument, Assumption and Evidence. Promoting Partnership for Health. London: Caipe, 2008.

5. Proch R. From silos to synergy: interprofessional education initiatives find warm welcome at Hopkins. Johns Hopkins Nursing. 2012; 10(1):33-35. [acesso em 2018 out 15]. Disponível em: https://magazine.nursing.jhu.edu/2012/04/from-silos-to-synergy/.

6. Peduzzi M. Equipe multiprofissional de saúde: conceito e tipologia. Rev. Saúde Pública. São Paulo 2001 fev; 35(1):103-109.

7. Telasai Júnior E. Práticas integrativas e complementares em saúde, uma nova eficácia para o SUS. Estud. av. São Paulo 2016 abr; 30(86):99-112.

8. Brasil. Ministério da Saúde. Secretaria de Atenção à Saúde. Política Nacional de Práticas Integrativas e Complementares no SUS. PNPIC-SUS. Brasília DF, Ministério da Saúde; 2006.

9. Galhardi WMP, Barros NF, Leite-Mor, et al. O conhecimento de gestores municipais de saúde sobre a Política Nacional de Prática Integrativa e Complementar e sua influência para a oferta de homeopatia no Sistema Único de Saúde local. Ciênc Saúde Colet. [internet]. 2013 [acesso em 2018 out 9]; 18(1):213-220. Disponível em: http://www.scielo.br/scielo.php?pid=s1413$-81232013000100022 \&$ script $=$ sci_abstract $\&$ tlng=pt.

10. Contatore OA, Barros NF, Durval MR, et al. Uso, cuidado e política das práticas integrativas e complementares na Atenção Primária à Saúde. Ciênc Saúde Colet. Rio de Janeiro. 2015 out; 20(10):32633273.

11. Sousa LA, Barros NF, Pigari JO, et al. Acupuntura no Sistema Único de Saúde: uma análise nos diferentes instrumentos de gestão. Ciênc Saúde Colet. Rio de Janeiro. 2017 jan; 22(1):301-310.

12. Losso LN, Freitas SFT. Avaliação do grau da implantação das práticas integrativas e complementares na Atenção Básica em Santa Catarina, Brasil. Saúde debat. Rio de Janeiro. 2017 set; 41(spe3):171-187.

13. Minayo MCS. Análise qualitativa: teoria, passos e fidedignidade. Ciênc. saúde coletiva. Rio de Janeiro. 2012; 17(3):621-626.

14. Barros NF, Fiuza AR. Evidence-based medicine and prejudice-based medicine: the case of homeopathy. Cad. Saúde Pública [internet]. 2014 [acesso em 2018 ago 9]; 30(11):2368-2376. Disponível em: https:// www.scielosp.org/scielo.php?pid=S010211X201400 1202368\&script=sci_arttext\&tlng=en.

15. Paradis E Whitehead CR. Louder than words: power and conflict in interprofessional education articles 1954-2013. Medical Education. Rockville. 2017 abr; 49(4):399-407.

16. Reis CR, Andrade SMO. Representação social do trabalho em equipe na atenção à mulher sob a ótica da enfermeira. Esc. Anna Nery, Rio de Janeiro. 2008 mar; 12(1):50-56. 
17. Marin MJS, Storniolo LV, Moravcik MY. Humanization of Care from the Perspective of the Family Health Strategy Teams in a City in the Interior of São Paulo, Brazil. Rev. Latino-Am. Enfermagem. Ribeirão Preto. 2010 jul-ago; 18(4):763-769.

18. Marqui ABT, Jahn AC, Resta DG, et al. Caracterização das equipes da Saúde da Família e de seu processo de trabalho. Rev. esc. enferm. USP. São Paulo. 2010; 44(4):956-961.

19. Moretti-Pires RO, Campos DA. Equipe multiprofissional em Saúde da Família: do documental ao empírico no interior da Amazônia. Rev. bras. educ. med. Rio de Janeiro. 2010; 34(3):379-389.

20. Araujo MBS, Rocha PM. Trabalho em equipe: um desafio para a consolidação da estratégia de saúde da família. Ciênc. saúde colet. Rio de Janeiro. 2007; 12(2):455-464.
21. Oliveira EM, Spiri WC. Programa Saúde da Família: a experiência de equipe multiprofissional. Rev. Saúde Públ. São Paulo. 2006 40(4), 727-733.

22. Peres CRFB, Caldas Júnior AL, Silva, RF, et al. O agente comunitário de saúde frente ao processo de trabalho em equipe: facilidades e dificuldades. Rev. esc. enferm. USP. São Paulo. 2011; 45(4):905-911.

23. Pereira RCA, Rivera FJU, Artamann E. O trabalho multiprofissional na estratégia saúde da família: estudo sobre modalidades de equipes. Interface (Botucatu). Botucatu. 2013; 17(45):327-340.

24. Aoandasan I, Reeves S. Key elements of interprofessional education. Part 2: Factors, processes and outcomes. J. Interprof. Care. London. 2005; 19:39-48.

Recebido em 30/05/2018

Aprovado em 28/08/2018

Conflito de interesses: inexistente

Suporte financeiro: não houve 